\title{
Stockage de déchets radioactifs dans l'argile
}

\section{G. ROUSSET ${ }^{*}$}

EDF-DER, Département MTC Route de Sens, 77250 Moret-sur-Loing

\section{A. GIRAUD*}

École Nationale Supérieure de Géologie, Laboratoire de

Géomécanique, BP 40, 54501 Vandœuvre-lèsNancy Cedex
Le chargement thermo-mécanique subí par le champ proche d'un stockage de déchets nucléaires dans I'argile est de grande ampleur; il se compose de deux chargements très distincts :

- le creusement (chargement mécanique) qui peut conduire à des convergences des ouvrages importantes de l'ordre de plusieurs pourcents:

- le chauffage (chargement thermique) causé par les déchets de type $C$; contrairement au creusement, ce dernier chargement produit de très faibles déformations puisque le déchet est bloqué dans le massif.

Dans cet article, on illustre dans le cas de l'argile de Mol cette dualité dans le chargement et ses effets sur le comportement à long terme des ouvrages.

Par ailleurs, on quantifie, pour cette argile, les couplages thermo-hydro-mécaniques (THM) à considérer pour le champ proche d'une part et pour le champ lointain d'autre part.
- Anciennement au Groupement pour l'étude des Structures Souterraines de Stockage - G. 3S-LMS (URA 317), École polytechnique 91128 Palaiseau Cedex.
For an underground disposal of radioactive wastes in a clayey formation, the thermo-mechanical loading is strong. It is made of two different loadings:

- the digging phase (mechanical loading) that can lead to an important closure of the galleries (several percents);

- the heating phase (thermal loading) due to $\mathrm{C}$ wastes disposal. In this paper, the effects of this double loading on the long term behavior of the underground repository are studied.

In case of Mol clay, thermo-hydro-mechanical couplings (THM) happening in the near field and the far field of a repository are quantified. 


\section{Introduction}

Dans certains projets de stockage de déchets radioactifs de catégorie C (déchets exothermiques), on prévoit de disposer les conteneurs cyclindriques dans l'alignement les uns des autres sur des longueurs variant de 10 à $100 \mathrm{~m}$ par exemple, à l'horizontale entre deux galeries ou à la verticale dans des petits puits ou bures creusés à partir de galeries.

Dans le cas d'un stockage dans l'argile, pour des questions de faisabilité et de réversibilité, il faudra certainement chemiser les ouvrages avant d'y mettre les déchets. Ainsi, le «chargement» thermo-mécanique en champ proche sera constitué des étapes suivantes:

- creusement, pose d'un soutènement (quelques jours) et attente (quelques mois);

- chauffage correspondant à la pose des conteneurs (plusieurs siècles).

Ce double chargement est spécifique du problème posé: chargement mécanique très ample suivi d'un chargement purement thermique.

L'objet de cet article est d'étudier les effets thermohydro-mécaniques (THM) de ce chargement sur le massif argileux à proximité des déchets (champ proche), puis en champ lointain.

Référence sera souvent faite à l'argile de Boom, présente à Mol (Belgique), puisque de nombreuses expériences géomécaniques y ont été conduites au cours des dix dernières années, grâce en particulier aux programmes européens de la CCE.

Les travaux qui ont nourri cette synthèse ont èté réalisés par le Centre d'Études Nucléaires belge (CEN/SCK; voir Beaufays et al., 1994) ou grâce à des thèses soutenues récemment au Groupement pour l'étude des Structures Souterraines de Stockage (G. 3S à l'École polytechnique: voir Rousset, 1988; Djéran, 1991; Giraud, 1993; Picard, 1994; Wong, 1994).

Tous ces travaux, dont la plupart ont été soutenus par l'ANDRA et la CEE, ont été publiés et sont donnés dans les références. Avant d'étudier le comportement des structures souterraines à Mol, nous donnons tout d'abord quelques indications sur le comportement thermo-hydro-mécanique de l'argile de Boom grâce à l'analyse de quelques essais originaux.

\section{2}

\section{Comportement de l'argile de Boom}

L'argile de Boom est une argile plastique dont la teneur en eau à saturation w varie sur le site de Mol entre 20 et $28 \%$ (moyenne $23 \%$ ). Il s'agit donc d'une argile très poreuse; sa "résistance mécanique ») est néanmoins plus élevée que celle d'un sol argileux: pour $w=23 \%$, la cohésion non drainée (Cu au sens de la mécanique des sols) est de l'ordre de $1 \mathrm{MPa}$.

Sous confinement, même faible, à l'essai triaxial. la localisation de la déformation ( $($ rupture $)$ ) survient pour des valeurs assez élevées de la déformation axiale, de l'ordre de $5 \%$. La figure 1 (essai triaxial non drainé cyclique à vitesse de déformation axiale imposée à $0,1 \% / \mathrm{min}$ ) montre que la majeure partie de la déformation est irréversible. En conséquence, le module d'Young (pente des droites de charge-décharge) est élevé, de l'ordre de $1000 \mathrm{MPa}$.

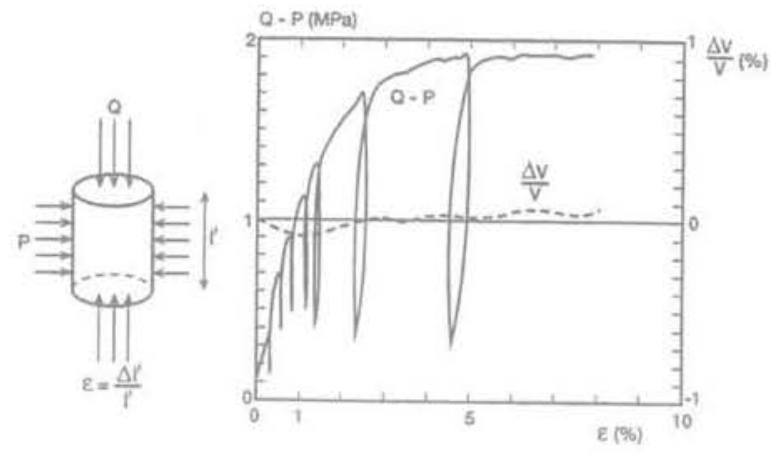

FG.1 Essai triaxial Cu cyclique sur l'argile de Boom.

Cyclic triaxial test $(\mathrm{Cu})$ on Boom clay.

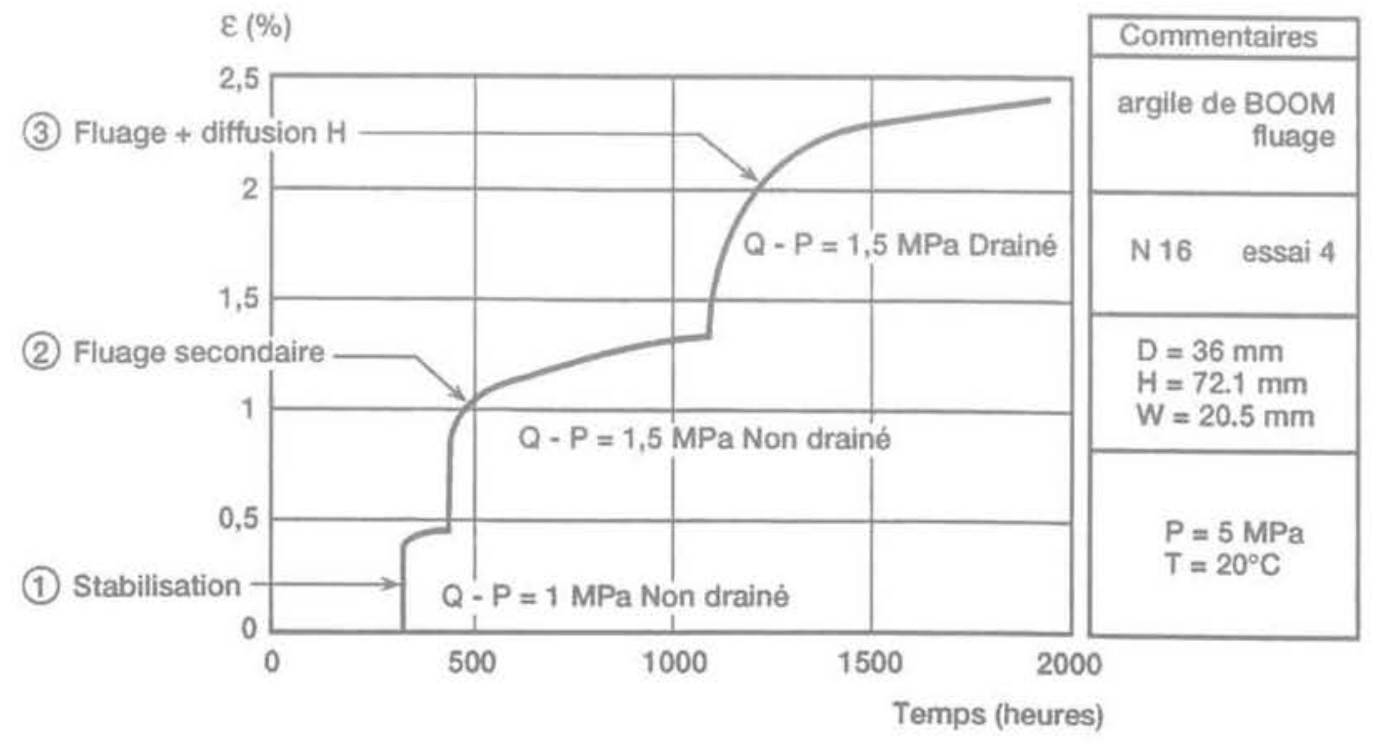

FIG.2 Essai de fluage sur l'argile de Boom.

Creep test on Boom clay. 
La partie quasi linéaire de la courbe entre 0 et $2 \%$ ne correspond donc pas à l'élasticité mais à un écrouissage positif du matériau.

Les effets différés sont très marqués sur ce matériau. Sur la figure 2, on donne les résultats d'un essai de fluage (confinement $\mathrm{P}=5 \mathrm{MPa}$, température ambiante) qui permet d'illustrer les principales caractéristiques du comportement différé de l'argile de Boom.

Pour une faible valeur du déviateur des contraintes (première partie de la courbe: phase non drainée avec un déviateur Q-P égal à $1 \mathrm{MPa}$ ), après une phase de fluage primaire, les déformations se stabilisent. Il existe donc un seuil viscoplastique non nul en deçà duquel il n'y a pas d'effets différés durables.

Au-dessus de ce seuil, il existe une phase de fluage secondaire, c'est-à-dire à vitesse constante, même en condition non drainée (partie 2 de la courbe).

Ces effets différés ne sont pas dus à la diffusion hydraulique puisque l'essai est non drainé, mais à la viscosité du matériau qui traduit sa capacité à se déformer au cours du temps sous contrainte uniforme constante et pression uniforme.

En conditions de drainage (partie 3 ), se superpose une déformation différée due à la diffusion de la pres- sion interstitielle. La constante de temps de ce phénomène est de l'ordre de quelques jours.

L'essai de relaxation par paliers donné sur la figure 3 permet d'illustrer d'une autre façon l'ampleur des effets différés. On a, pour $\mathrm{P}=3 \mathrm{MPa}$, tracé la courbe $(\mathrm{Q}-\mathrm{P})(\varepsilon)$ à court terme (l'enveloppe supérieure de la courbe) et à long terme (courbe qui joint les points correspondant à la stabilisation pour chaque palier). On remarque de plus sur la courbe $(\mathrm{Q}-\mathrm{P})$ ( $\mathrm{t}$ ) que la constante de temps des effets différés est de l'ordre de la journée.

Au cours de cet essai, la perméabilité a été mesurée également. Les deux courbes croissantes en fonction du temps de la figure 3 sont les volumes cumulés $V_{B}$ et $V_{H}$ d'eau injectée en partie basse (pression d'eau = $2 \mathrm{MPa}$ ) et récupérée en partie haute (pression d'eau = $1,7 \mathrm{MPa}$ ). On constate que le débit mesuré ne dépend pas de l'état de plastification du matériau. Il vaut environ $4 \mathrm{~mm}^{3} / \mathrm{h}$, ce qui correspond, si l'on fait l'hypothèse de la loi de Darcy (gradient $i=420$ ), à une perméabilité de:

$$
\mathrm{K}_{\mathrm{h}}=2,710^{-12} \mathrm{~m} / \mathrm{s}
$$

Nous allons retrouver ces propriétés dans le comportement des ouvrages in situ, thème du paragraphe suivant.
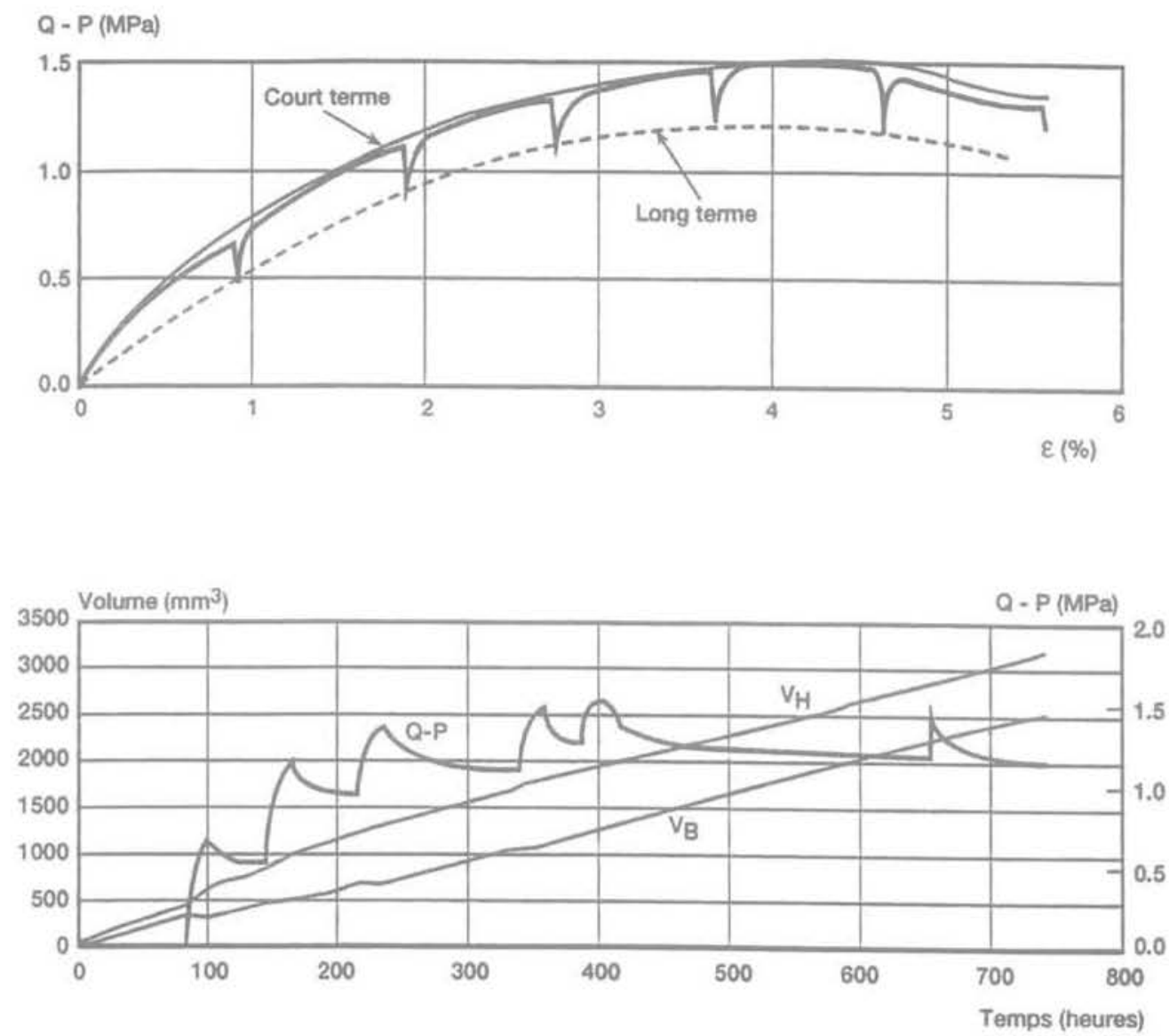

FIG. 3 Essai de relaxation par paliers sur l'argile de Boom. Relaxation test on Boom clay. 


\section{Le champ proche}

\section{1}

\section{Le chargement mécanique: couplages HM}

A court terme, les ouvrages " galeries horizontales $)$ et "puits verticaux») présentent la même spécificité du point de vue mécanique et hydraulique. En effet, la perturbation majeure à ce niveau correspond aux phases de creusement et de pose des soutènements ou des tubes. Localement, c'est au cours de cette phase, c'està-dire à court terme (échelle de la journée), que le massif en champ proche subit le chargement le plus violent.

Les différences essentielles entre ces deux types d'ouvrage sont la direction (horizontale ou verticale) et le diametre (de $50 \mathrm{~cm}$ pour les puits de stockage des verres à quelques mètres pour les galeries de manutention). Ces deux paramètres ont peu d'influence sur le comportement qualitatif des ouvrages souterrains en général. Il n'est donc pas nécessaire, à ce niveau de lanalyse, de distinguer les tunnels des puits.

L'étude du cas de Mol permet d'illustrer les différentes particularités du champ proche. La figure 4 donne l'emplacement de divers ouvrages du site. La "petite galerie» (point 1) a en particulier permis de mesurer in situ les déplacements en champ proche (mesures extensométriques).

La figure 5 donne l'évolution du déplacement radial de cinq points du massif situés à des distances croissantes de la paroi de la galerie.

L'extensomètre a été placé avant la réalisation de la "petite galerie», ce qui permet de mesurer les déplacements à partir du massif vierge, mesure très précieuse et très rare en géotechnique (Rousset, 1988).

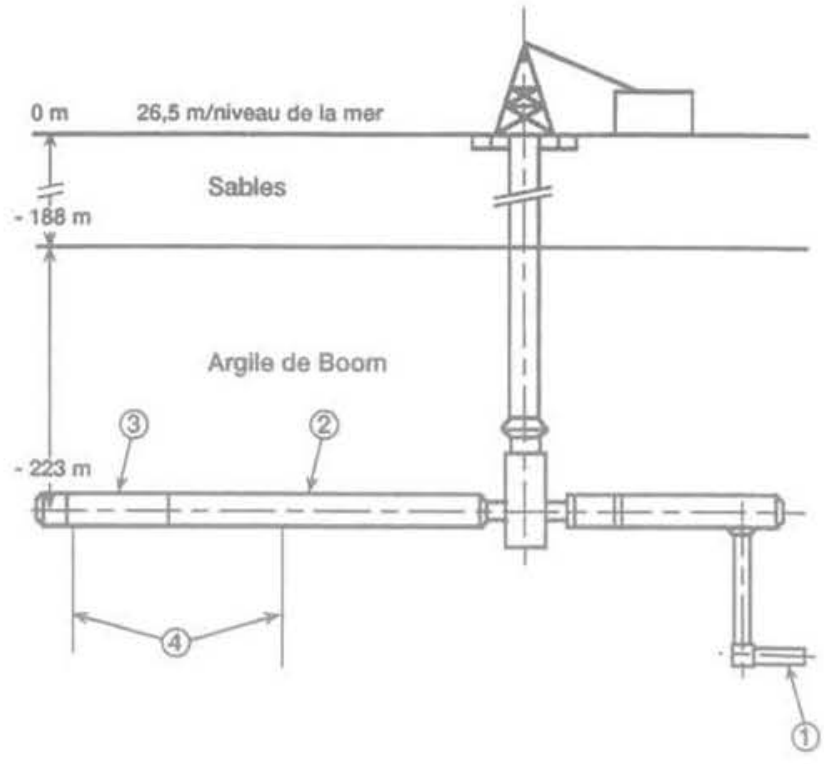

FG.4 Les ouvrages souterrains à Mol. Underground facilities at Mol.

Les portions en traits gras de ces courbes correspondent à des phases de creusement effectif (avancement du front de taille) : la réponse du massif (déplacements radiaux d'autant plus importants que le point de mesure se situe près de la paroi) est instantanée.

Ces mouvements de convergence se produisent avant même que le front de taille ne passe sous les capteurs, c'est-à-dire bien avant que le soutènement, constitué ici de claveaux de béton, ne puisse être posé.

Les portions en traits fins se réfèrent à des phases d'arrêt de chantier; on constate que les mouvements de convergence se produisent encore, mais à des vitesses plus lentes. Ce comportement différé à court
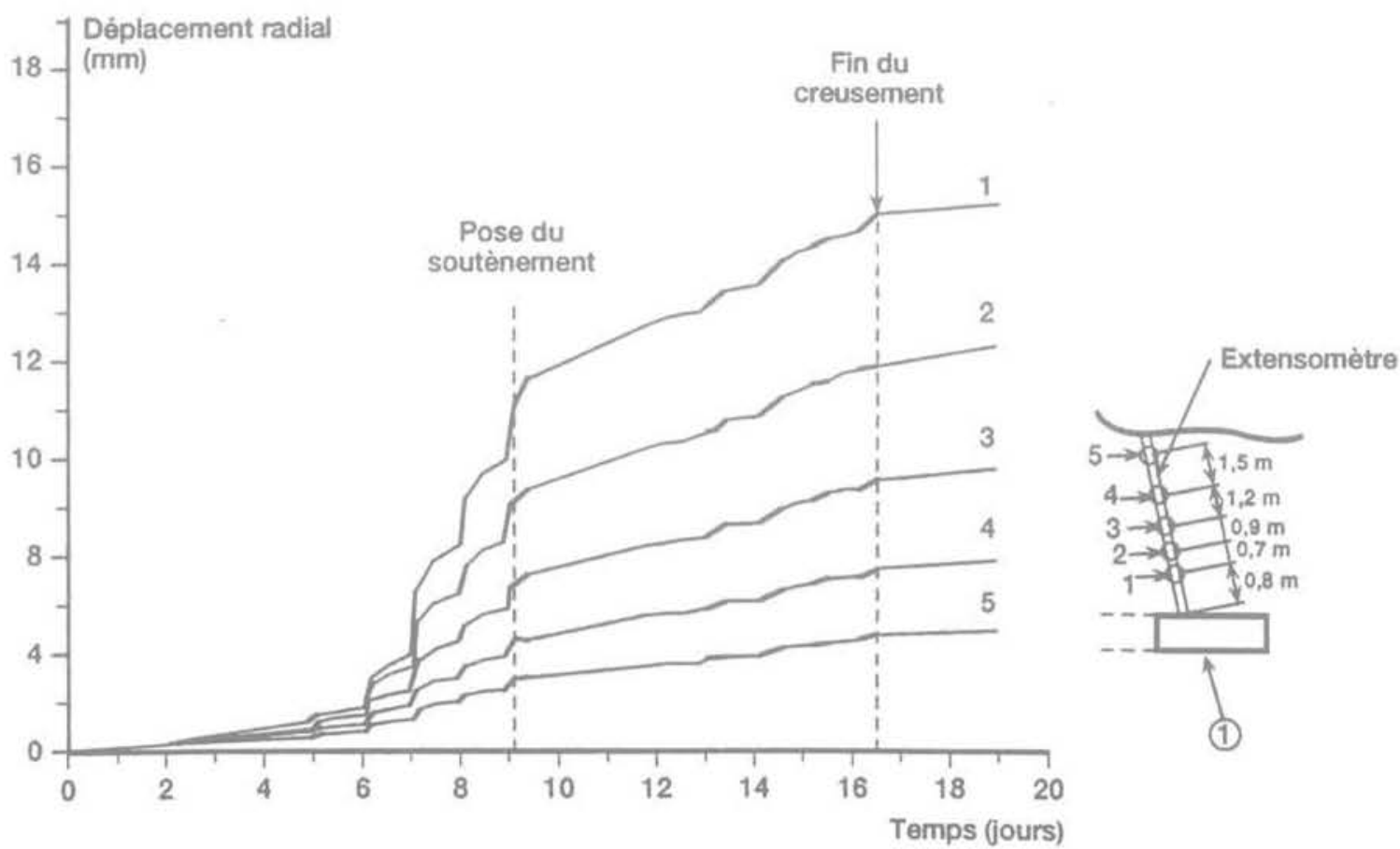

FG. 5 Mesures extensométriques: influence du creusement sur la convergence de la galerie. Extensometric measurements. 


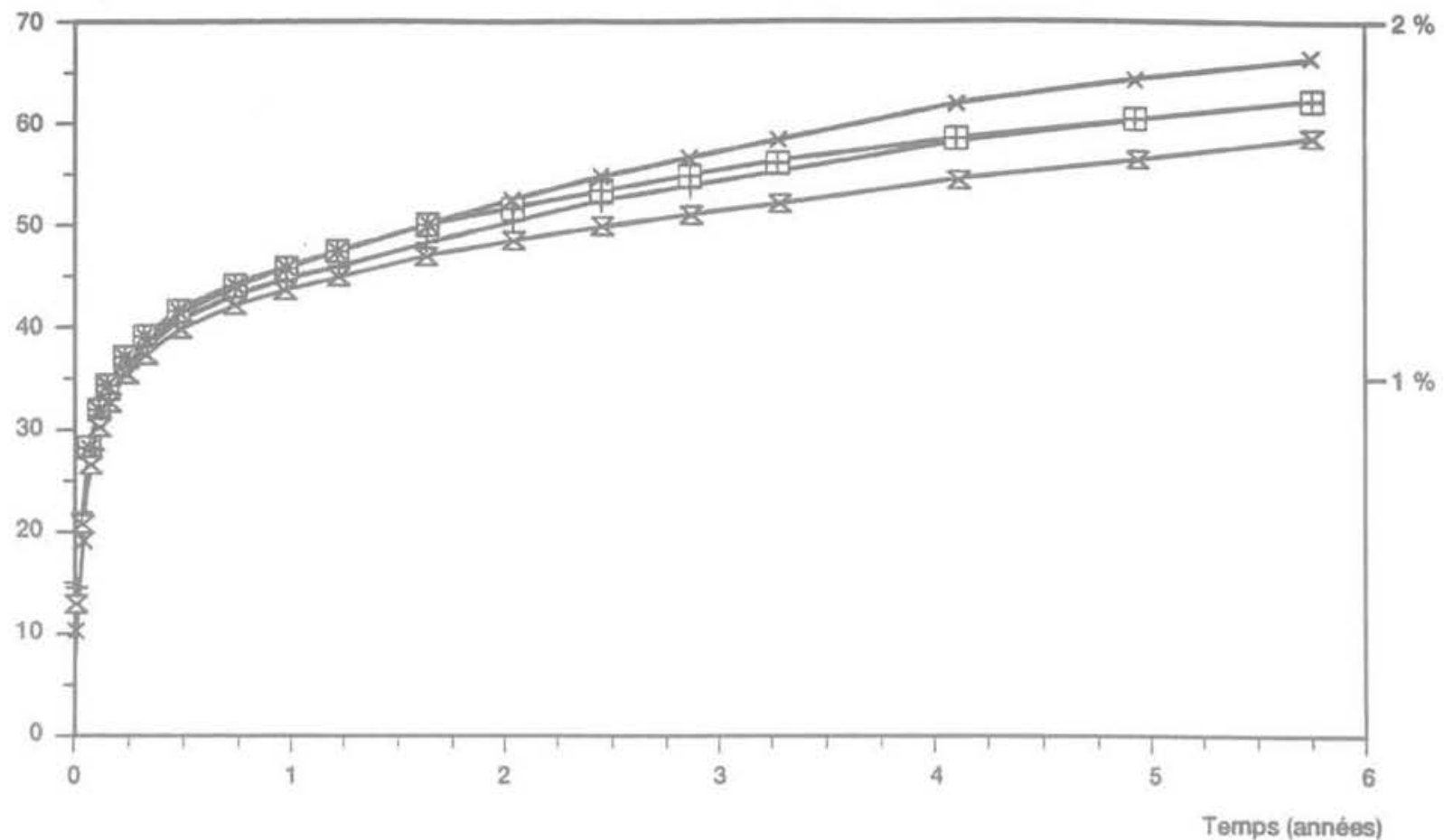

FG.6 Convergence du soutènement du test drift (Beaufays et al., 1994). Closure of the test drift lining.

terme est dû à la fois au phénomène de diffusion hydraulique (la galerie agit comme un drain dans un milieu où la pression hydraulique vaut initialement $2 \mathrm{MPa}$ environ) et aux propriétés de fluage de l'argile (Rousset et al., 1993).

A l'issue du creusement, l'ensemble de ces mouvements radiaux a produit une convergence totale $U$ de plus de $8 \%$ (U, est le rapport entre le déplacement d'un point de massif situé au niveau de la paroi et le rayon initial de la galerie).

Cette valeur est quantitativement très importante et correspond à des mouvements irréversibles du terrain: une zone plastique s'est fortement développée autour de la galerie, bien que le soutènement ait été posé très rapidement aussi près du front de taille que possible.

Autrement dit, le creusement du massif, malgré la présence d'un soutènement, correspond à un chargement mécanique de grande ampleur.

Sur la figure 6, on a reporté la convergence du soutènement du test drift (point 2 sur la figure 4; voir Beaufays et al.., 1994), qui est une galerie semblable à la précédente. La seule différence notable porte sur le diamètre ( $3,5 \mathrm{~m}$ utiles au lieu de $1,4 \mathrm{~m})$.

On constate que la convergence évolue lentement, toujours en raison de la diffusion hydraulique et du comportement différé de l'argile de Boom; mais que son ampleur est plus faible.

La convergence du soutènement à long terme ne représente qu'une faible partie de la convergence totale de la paroi, la plus grosse partie se produisant lors du creusement.

Néanmoins, ces effets différés sont sensibles sur une très longue période. Le cas de la galerie cintrée (point 3 de la figure 4; voir sur la figure 7, l'évolution de la convergence de ce soutènement coulissant) est tout à fait démonstratif: après cinq ans, les mouvements du massif se produisent toujours ce qui laisse penser que l'équilibre final ne sera atteint qu'après quelques dizaines d'années. Cet équilibre se produira nécessairement car, conformément aux essais du type de celui représenté sur la figure 2 , on démontre en laboratoire qu'il existe un seuil non nul du déviateur des contraintes en deçà duquel il n'y a plus d'effets différés (propriété de résistance à long terme des argiles en général).

Après cinq ans, la convergence moyenne de ce soutènement particulier est égale à 2,5\% alors que l'on peut estimer que la convergence à la paroi avant la pose du soutènement est de l'ordre de $5 \%$ (Bernaud et Rousset, 1993).

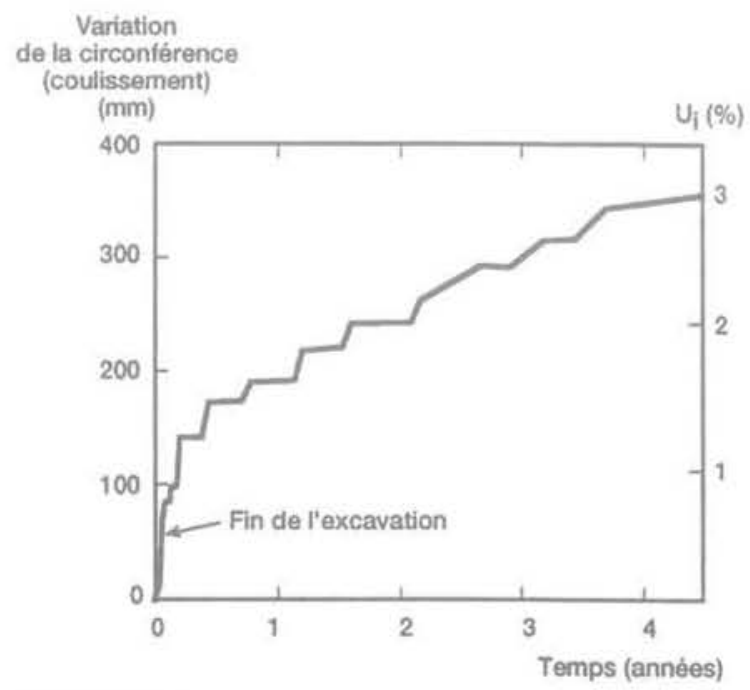

FG, 7 Évolution de la convergence de la galerie cintrée.

Closure of the rib lined gallery. 


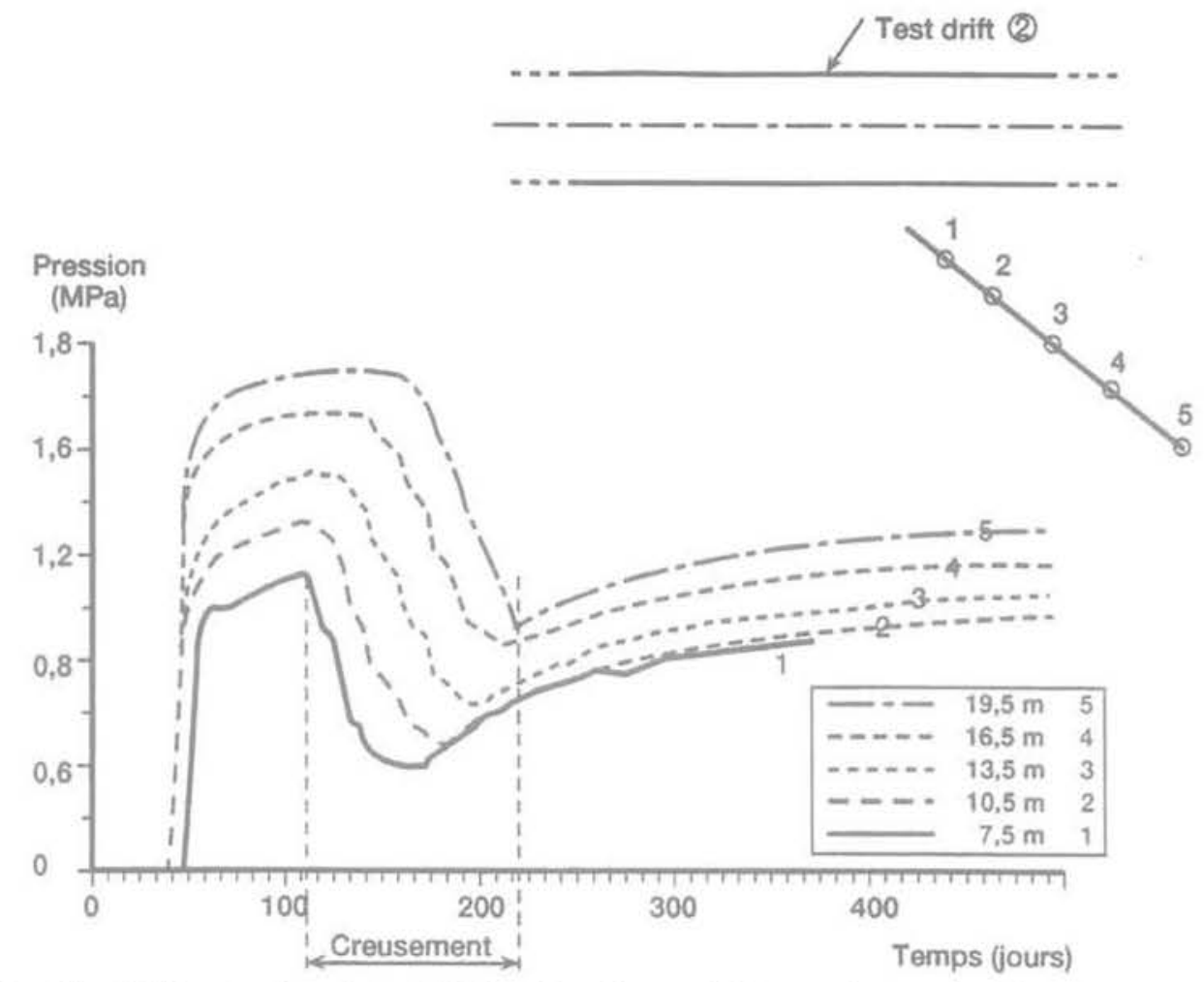

FG. 8 Couplage HM : pressions interstitielles dans le massif autour du test drift (Beaufays et al., 1994). Hydro-Mechanical coupling : interstitial water pressures within the rock-mass.

Une autre mesure très intéressante autour du test drift est la pression interstitielle dans le massif. Sur la figure 8, sont représentées les évolutions de la pression en cinq points différents du massif, placés comme indiqué sur le petit schéma de la figure 8 (mesures du CEN/SCK; voir Beaufays et al., 1994).

Ces courbes sont composées de trois parties:

- au début, il n'y a pas d'évolution: avant le creusement du test drift, la pression est stable (elle n'est par contre pas homogène, certainement en raison de la présence voisine de plusieurs ouvrages qui jouent un rôle de drain):

- pendant le creusement, les pressions interstitielles chutent fortement: le couplage HM est donc très fort. II s'agit bien d'un couplage (influence directe et instantanée de la variation de déformation sur la pression) et pas seulement d'un effet de diffusion hydraulique;

- après le creusement, les pressions augmentent lentement pour tendre par diffusion vers un état asymptotique différent de l'état initial, en raison de l'effet de drain du test drift.

La constante de temps caractéristique de ce phénomène différé est de l'ordre du mois, comme le prévoient d'ailleurs les analyses simples de diffusion hydraulique pour un modèle de comportement élastique avec un écoulement obéissant à la loi de Darcy (voir annexes 1 et 2).

\section{2}

\section{Le chargement thermique: couplages THM}

Les déchets radioactifs de type $\mathrm{C}$ dégagent de la chaleur, ce qui va faire augmenter la température dans le massif en champ proche. Il est aujourd'hui commu- nément admis que le seul mode de transmission de la chaleur est la diffusion (Djéran et al., 1993); cette diffusion n'est pas couplée aux aspects $\mathrm{M}$ et $\mathrm{H}$ dans le cas des argiles. Autrement dit, le champ thermique peut être calculé indépendamment du comportement hydraulique et mécanique.

Pour l'argile de Boom, la constante de temps de diffusion thermique est du même ordre de grandeur que sa constante hydraulique, de l'ordre du mois pour le champ proche.

Dans «l'autre sens», en revanche, les couplages sont importants: la température induit des contraintes et déformations thermiques; de même, l'eau se dilatant plus que le matériau, le champ de température va conditionner largement le champ de pression.

Une caractéristique importante de ce chargement est qu'il ne produit que des déformations très petites (de l'ordre de $0,1 \%$ ), contrairement à la phase de chargement mécanique (creusement) pour laquelle on a vu que l'ordre de grandeur des déformations était 10 à 100 fois plus grand.

Autrement dit, le couplage THM complet ne nous intéresse que dans une gamme de déformations très petites (ce qui simplifie l'analyse); par contre, ces déformations d'origine thermique se produisent à partir d'un état initial déjà plastifié par le chargement précédent; en particulier, on notera que cet état est hétérogène (le champ de contrainte autour d'un puits dépend, en effet, très fortement de la distance du point d'analyse l'axe du puits).

L'essai CACTUS réalisé à Mol (Picard et al., 1994) a permis d'étudier l'ensemble de ces couplages et de mettre l'accent sur la particularité essentielle du chargement en champ proche: le chargement mécanique (sans thermique) à court terme suivi du chargement thermique (sans déformation importante). 
L'essai CACTUS (Fig. 9; point 4 sur la figure 4) consiste en deux sondes thermiques (diamètre $30 \mathrm{~cm}$, longueur $4 \mathrm{~m}$ ) placées à Mol dans des puits de $15 \mathrm{~m}$ de longueur réalisés à partir du test drift (profondeur $250 \mathrm{~m}$ ); l'espace annulaire entre la paroi du massif et les sondes est rempli de boue (matériau fluide) faite à partir d'argile du site.

Sur la figure 10 (température en fonction du temps), on mesure en particulier la constante de temps du problème thermique, de l'ordre de 1 mois.

La figure 11 donne l'évolution du champ de contrainte radiale, oủ l'on distingue bien les phases de creusement, de restauration des contraintes (effets différés), puis de chauffage et enfin de refroidissement.

On notera qu'il faut environ 6 mois pour que la stabilisation mécanique et hydraulique au niveau du puits soit atteinte.

En résumé, pour ce qui concerne le champ proche dans le cas de $\mathrm{Mol}$ on retiendra les points clés suivants :

- zone plastique créée dès le creusement (ordre de grandeur de la convergence $5 \%$ ), donc zone durablement "perturbée» avant la mise en place des colis;

- effets différés hydro-mécaniques importants (ordre de grandeur de la durée: quelques mois pour les puits, quelques années pour les ouvrages de grand diamètre);

- chargement thermique non accompagné de déformations importantes;

- couplages THM forts (ordre de grandeur du temps de diffusion thermique et hydraulique: quelques mois).

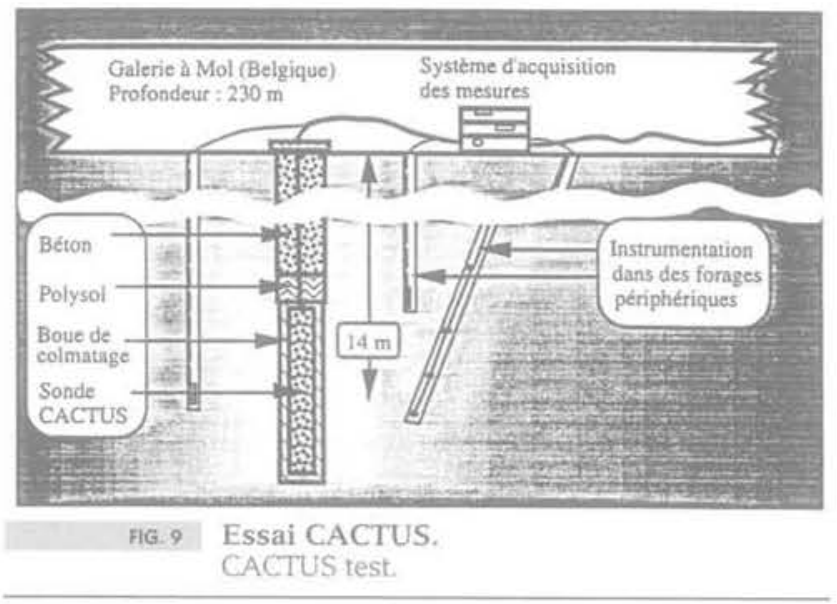

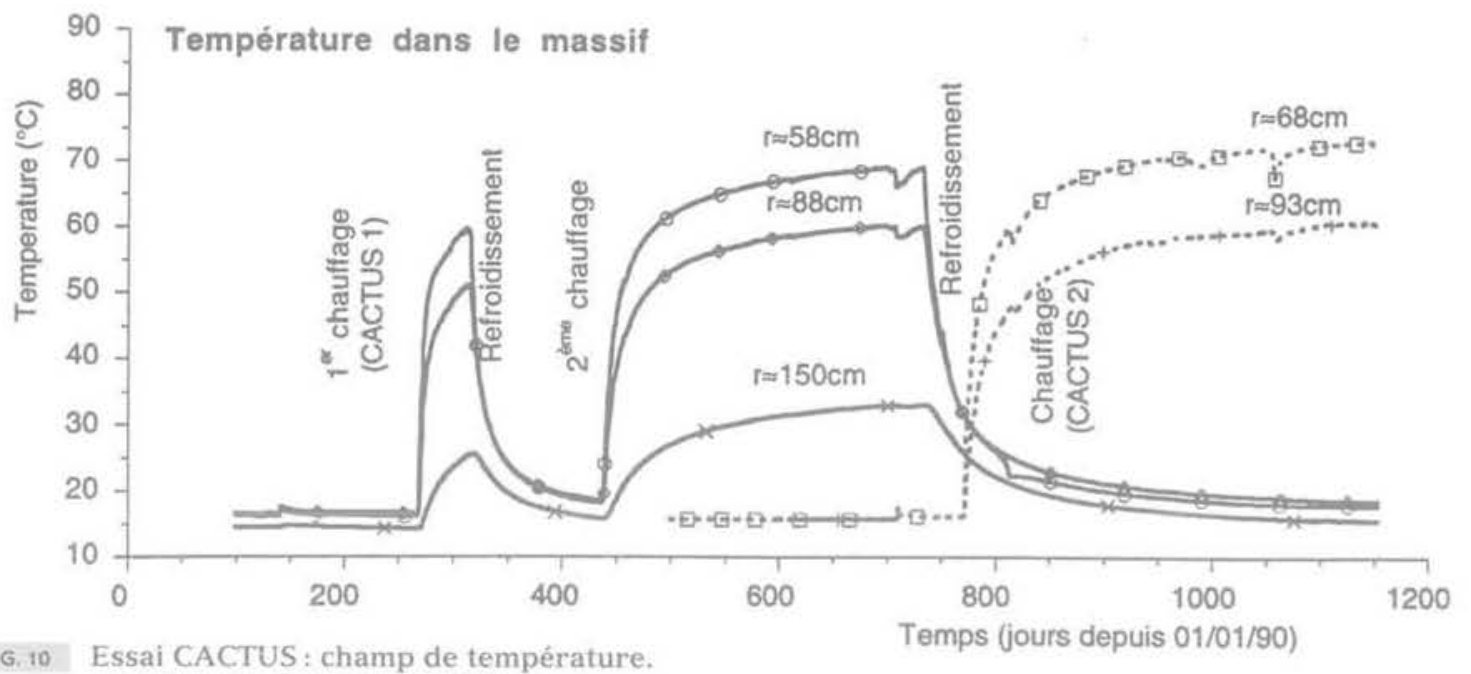

FG. 10 Essai CACTUS : champ de température. CACTUS test: temperature field.

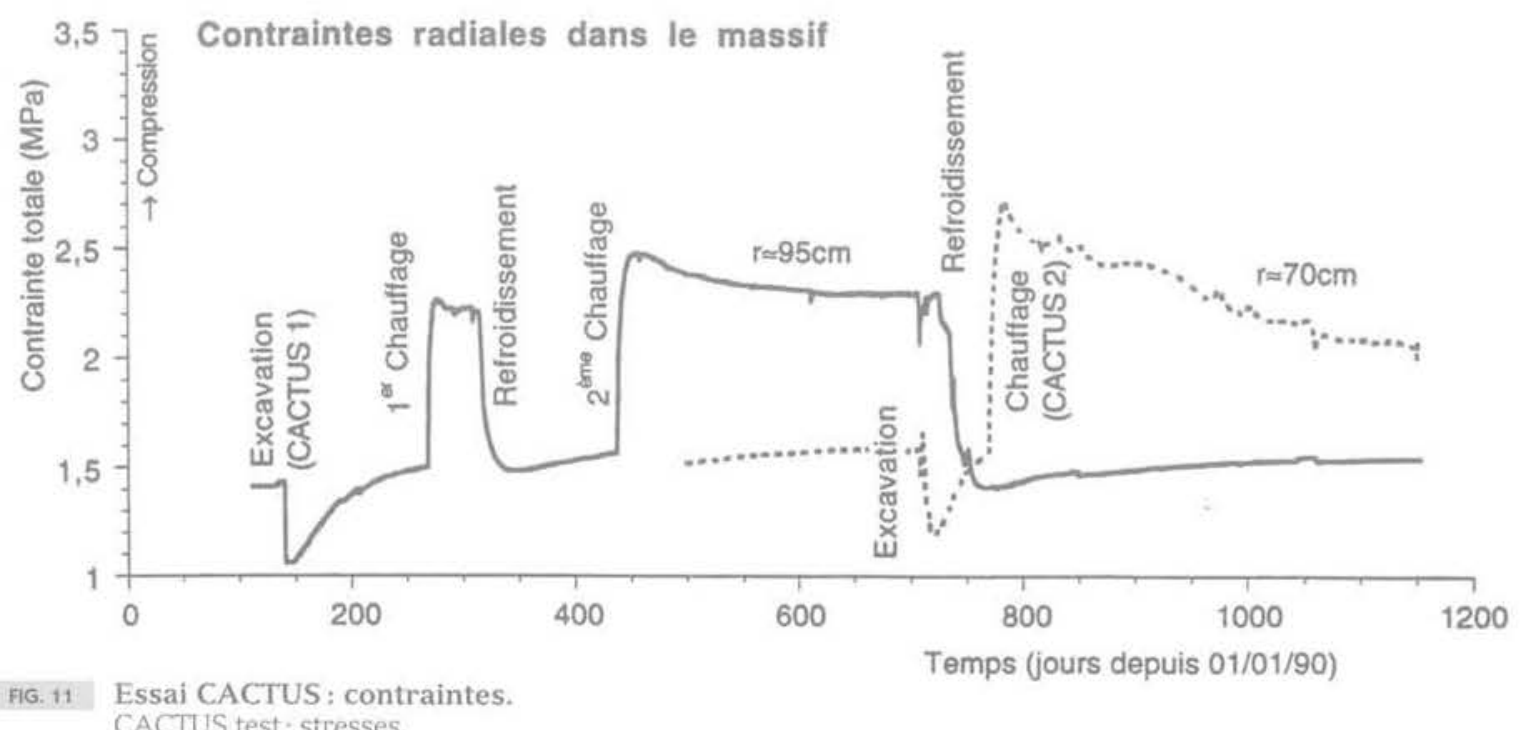




\section{Le champ lointain}

Le champ lointain concerne l'ensemble de la roche hôte ainsi que les autres couches géologiques.

Le champ lointain est lui aussi soumis à un chargement mécanique qui correspond à la réalisation des ouvrages souterrains lesquels, du point de vue mécanique, induisent un déconfinement global du terrain et du point de vue hydraulique jouent le rôle de drain.

Une différence essentielle avec le champ proche est que le chargement thermique peut être considéré comme simultané au chargement mécanique (cas du remplissage progressif des galeries).

La constante de temps de ce chargement est plus grande que celle du champ proche, de l'ordre de plusieurs années.

Pour ce qui concerne l'effet du chargement thermique, les figures 12 à 15 illustrent le cas simplifié unidimensionnel d'un stockage de dimensions latérales infinies dans un massif homogène, isotrope et linéairement élastique (Giraud et Rousset, 1995).

On a négligé le chargement dû aux ouvrages (déconfinement) et on a porté sur les figures les variations des divers paramètres à partir d'un état initial nul (température, pression et contraintes principales). Grâce au principe de superposition, pour obtenir les valeurs réelles des paramètres, il suffit d'ajouter leurs valeurs initiales aux variations calculées.

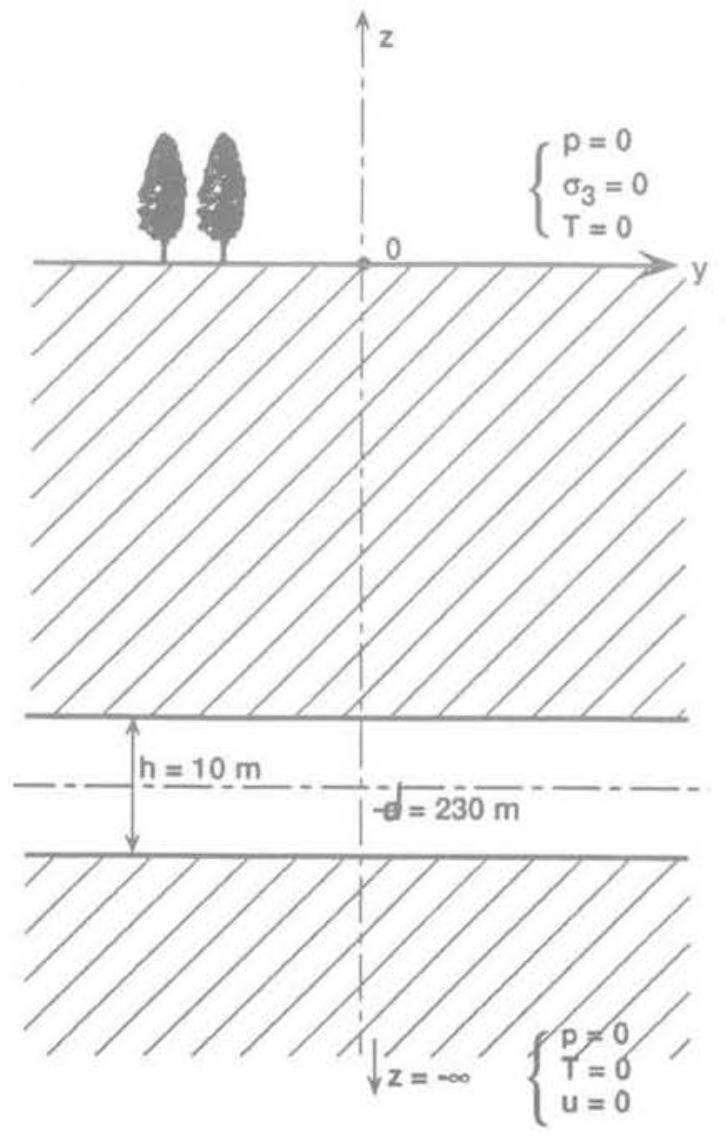

FG. 12 Géométrie en champ lointain - Paramètres. Far field geometry - Data.

La source de chaleur est supposée uniformément répartie dans les 10 mètres d'épaisseur du stockage.

Les valeurs numériques des treize paramètres que comprend le modèle (les neuf paramètres intrinsèques du problème THM linéarísé, dont les équations sont données en annexe 2, deux paramètres pour la géométrie du stockage: profondeur du centre et épaisseur du stockage, deux parametres pour la puissance thermique $Q_{0} e^{-w t}$ : puissance volumique initiale $Q_{0}$ et

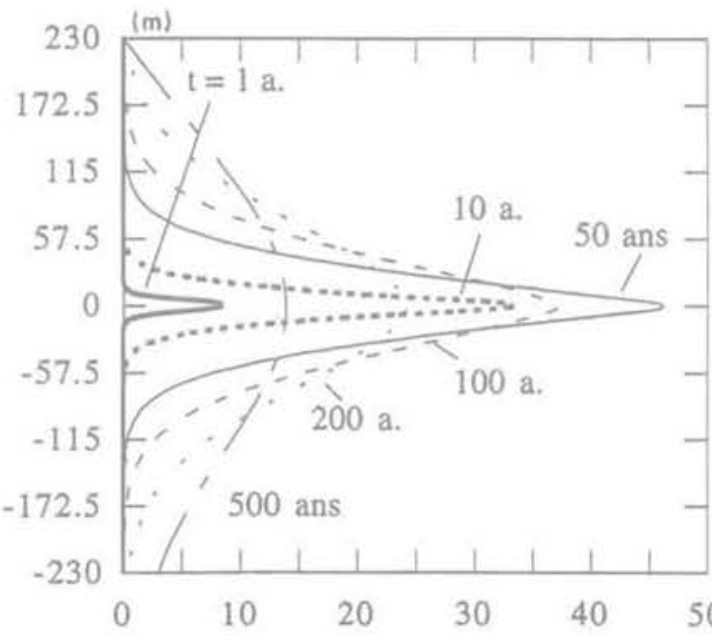

$\left({ }^{\circ} \mathrm{C}\right)$

FIG. 13 Élévation de température en champ lointain.

Increase of temperature for the far field.

\section{Module de compressibilité drainé $K_{0}$ (MPa)}

Module de cisaillement $\mu(\mathrm{MPa})$

Coefficients de Biot M (MPa) b

$$
\text { ( }
$$

Dilatations thermiques

$$
\begin{array}{ll}
\alpha_{0} & \left(K^{-1}\right) \\
\alpha & \left(K^{-1}\right)
\end{array}
$$

\section{Conductivité thermique} $\mathrm{K}_{\mathrm{T}}(\mathrm{W} / \mathrm{m} / \mathrm{K})$

Chaleur spécifique

$$
\mathrm{C}_{\varepsilon}^{\circ}\left(\mathrm{J} / \mathrm{m}^{3} / \mathrm{K}\right)
$$

Perméabilité $K_{h}(\mathrm{~m} / \mathrm{s})$

Epaisseur du stockage $\mathrm{h}(\mathrm{m})$

Profondeur du stockage $d(m)$

Puissance volumique initiale $Q_{0}\left(W / m^{3}\right)$

\section{Décroissance radioactive} W $\left(a n^{-1}\right)$

\section{0}

60

5500

1

$10^{-5}$

$4,3610^{-5}$

1,7

$2,8510^{6}$

$410^{-12}$

10

230

1

0,024 

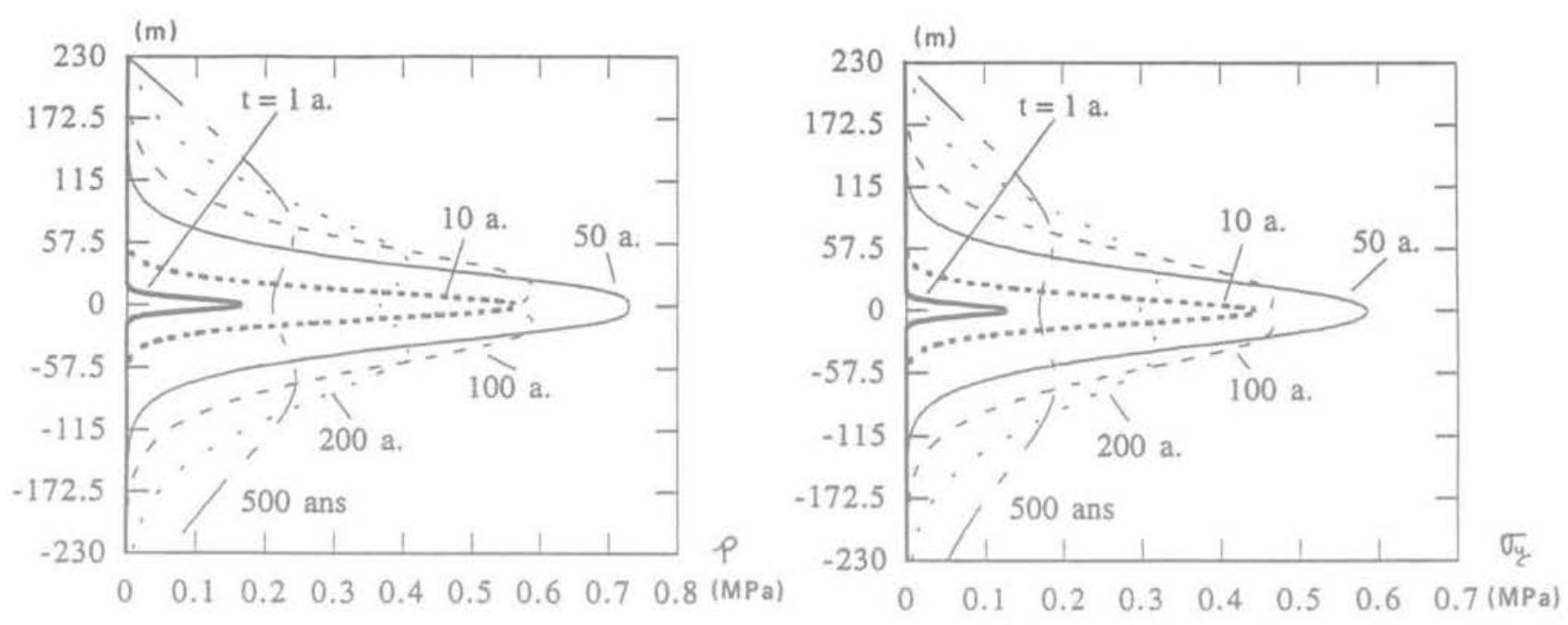

916. 14 Pression et contrainte horizontale.

Pressure and horizontal stress.

décroissance radioactive w) sont données dans le tableau de la figure 12. Elles correspondent au cas d'une argile relativement compressible du type de celle de Mol.

Le paroxysme thermique est atteint au bout d'une cinquantaine d'années (Fig. 13). L'augmentation de pression interstitielle, du même ordre de grandeur que la pression initiale, intéresse une forte épaisseur du terrain autour du stockage (Fig. 14). Le soulèvement de la surface du sol dû à la dilatation des terrains n'est maximal qu'au bout de 200 ans (Fig. 15).

Dans ce modèle simple, la contrainte verticale reste constante au cours du chargement (sa valeur absolue est égale au "poids des terres» comme on s'en convainc aisément en étudiant l'équilibre d'une colonne de sol).

Le déviateur maximal des contraintes est donc égal à la valeur absolue de la variation de la contrainte horizontale (Fig. 14).

Il est intéressant de constater que ce déviateur ne dépasse pas $1 \mathrm{MPa}$ au cours du chargement thermique, valeur de l'ordre de grandeur de la cohésion C du matériau.

On en déduit que, contrairement au changement mécanique en champ proche, le chargement thermique en champ lointain ne devrait pas conduire à un développement important de la plasticité dans le massif. Ceci justifie a posteriori le choix d'un modèle poro-élastique pour étudier le champ lointain.

Le couplage THM en champ lointain est fort pendant plusieurs siècles puisqu'il faut attendre 200 ans avant que la chaleur commence à se dissiper par convection depuis la surface du sol (voir aussi Ould Amy et Rousset 1995, pour le cas plus complexe de la "galette » circulaire traitée en axisymétrie).

\section{5}

\section{Conclusion}

Le stockage de déchets radioactifs exothermiques dans l'argile correspond à un double chargement mécanique et thermique dont les effets sont différents.

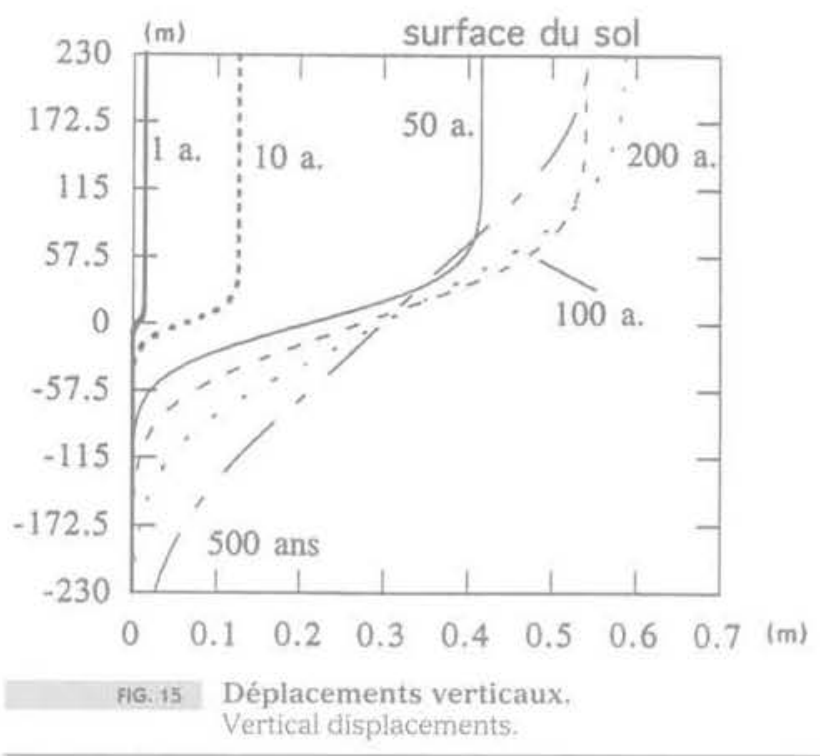

En champ proche, la construction des ouvrages (court terme) s'accompagne d'un déconfinement important et la plasticité se développe beaucoup. Ensuite, lorsque les déchets et le matériau de colmatage éventuel sont déposés, commence le chargement thermique qui crée des couplages THM extrêmement forts.

En champ lointain, l'effet du chargement mècanique est moins important qu'en champ proche et les déviateurs de contrainte thermique restent relativement faibles en valeur absolue ce qui signifie que le comportement du massif reste essentiellement réversible au cours de la phase thermique.

A l'avenir, en thermo-hydro-mécanique du champ proche, il serait intéressant de développer les recherches sur l'influence de la plasticité et de la température sur la perméabilité de l'argile.

Pour le champ lointain, il faudrait étudier en particulier les phénomènes de convection thermique dans les nappes aquifères ainsi que les effets de l'hétérogénéité et de l'anisotropie des géomatériaux. 


\section{Annexes}

\section{A.1 Les échelles d'espace et de temps} (Stockage de déchets nucléaires dans l'argile)

On distingue classiquement deux échelles d'espace, le champ proche (zones de dimensions métriques autour des ouvrages souterrains et des déchets) et le champ lointain (zone de dimensions hectométriques, à l'échelle du massif).

Le diagramme suivant illustre de façon simplifiée les diverses gammes de temps que l'on peut distinguer pour chacune des échelles d'espace ainsi que la nature des couplages les plus importants. On adopte les abréviations suivantes:
$T$ : Thermique
(diffusion de chaleur)
$\mathrm{H}$ : Hydraulique (diffusion de l'eau interstitielle)
M : Mécanique
C : Chimique
(transport et migration).

$$
k=\frac{K_{h}}{\rho_{f}^{0} g}
$$

$\left(\rho_{\mathrm{f}}^{\circ}\right.$ : masse volumique du fluide; $K_{\mathrm{h}}$ : perméabilité apparente; $\mathrm{g}$ : accélération de la pesanteur; $\mathrm{k}$ : conductivité hydraulique).

La conservation de la masse fluide:

$$
\dot{\mathrm{m}}+\operatorname{div} \underline{\mathrm{M}}=0
$$

fournit, grâce à (1), la première équation de diffusion du problème:

$$
\frac{\dot{\mathrm{m}}}{p_{\mathrm{n}}^{0}}=\mathrm{k} \Delta \mathrm{p}
$$

- Équation de diffusion de la chaleur

La loi de Fourier indique, quant à elle, que la relation entre vecteur courant de chaleur q et gradient de température est linéaire:

\begin{tabular}{|c|c|c|c|c|}
\hline & Champ proche & & Champ lointain & \\
\hline Jour & $\begin{array}{l}\text { - Chargement mécanique rapide } \\
\text { (creusement) }\end{array}$ & (HM) & & \\
\hline Mois & $\begin{array}{l}\text { - Réponse mécanique différée } \\
\text { - Paroxysme thermique }\end{array}$ & $\begin{array}{l}(\mathrm{HM}) \\
(\mathrm{THM})\end{array}$ & & \\
\hline Année & - Diffusions & (TH) & - Chargement mécanique & $(\mathrm{HM})$ \\
\hline Siècle & & & - Chargement thermique & (THM) \\
\hline Millénaire & - Corrosion, transport & $(\mathrm{HC})$ & - Diffusions & (TH) \\
\hline $10^{p}$ années & -id- & & - Migration des radioéléments & (HC) \\
\hline
\end{tabular}

$$
\underline{q}=-K_{T} \underline{\operatorname{grad} T}
$$

\section{A.2 Les équations de base du problème THM}

Nous rappelons dans cette annexe les équations complètes du problème THM le plus simple, c'est-àdire celui correspondant au comportement élastique linéaire d'un matériau homogène et isotrope avec écoulement obéissant à la loi de Darcy et diffusion thermique de Fourier (Biot, 1941; Coussy, 1991).

En thermo-poro-élasticité, les variables d'état sont:

$\underline{\underline{\varepsilon}}$ tenseur des déformations:

$\mathrm{m}$ apport de masse fluide par unité de volume; $\tau$ variation de température.

Les variables duales sont respectivement, le tenseur des contraintes $\underline{\underline{\sigma}}$, la pression du fluide $p$ et l'entropie S.

\section{- Équation de diffusion du fluide}

La loi de Darcy pour un matériau isotrope propose une correspondance linéaire entre le vecteur courant de masse fluide $\underline{M}$ et le vecteur gradient de pression:

$$
\frac{1}{\rho_{\mathrm{f}}^{0}} \underline{M}=-\mathrm{k} \underline{\operatorname{grad}} \mathrm{p}
$$

avec:
Si l'on appelle $\mathrm{r}$ la production volumique de chaleur, l'équation thermique s'écrit:

$$
\mathrm{T}_{0} \dot{\mathrm{S}}=\mathrm{T}_{0} \mathrm{~S}_{\mathrm{m}}^{0} \dot{\mathrm{m}}+\mathrm{r}-\operatorname{div} \underline{\mathrm{q}}
$$

( $S_{\mathrm{m}}^{\circ}$ : entropie massique initiale du fluide)

de sorte que l'équation de la chaleur (porter (4) dans (5)) s'écrit:

$$
\mathrm{T}_{0} \dot{\mathrm{S}}-\mathrm{T}_{0} \mathrm{~S}_{\mathrm{m}}^{0} \dot{\mathrm{m}}=\mathrm{K}_{\mathrm{T}} \Delta \mathrm{T}+\mathrm{r}
$$

\section{- Équation d'équilibre}

En l'absence de force de masse, l'équilibre mécanique s'écrit:

$$
\underline{\text { div }} \underline{\underline{\sigma}}=0
$$

où $\cong$ est le tenseur des contraintes.

\section{- Loi de comportement}

Pour un milieu isotrope avec un état initial naturel, il suffit de sept paramètres indépendants pour établir une relation linéaire entre $\sigma, \mathrm{p}, \mathrm{S}$ d'une part et $\varepsilon, \mathrm{m}, \tau$ d'autre part $\left(\tau=\mathrm{T}-\mathrm{T}_{0}\right)$ : 


$$
\begin{gathered}
\underline{\underline{\sigma}}=\lambda(\operatorname{tr} \underline{\underline{\varepsilon}}) \underline{\underline{1}}+2 \mu \underline{\underline{\varepsilon}}-\frac{\mathrm{bM}}{\rho_{0}^{\mathrm{f}}} \mathrm{m} \underline{\underline{1}}-3 \alpha \mathrm{K} \tau \underline{\underline{1}} \\
\mathrm{p}=\mathrm{M}\left(-\mathrm{b} \operatorname{tr} \underline{\underline{\varepsilon}}+\frac{1}{\rho_{0}^{i l}} \mathrm{~m}\right)+3 \alpha_{m} \mathrm{M} \tau \\
\mathrm{S}=\mathrm{m} \mathrm{S}_{\mathrm{m}}^{0}+3 \alpha_{0} \mathrm{~K}_{0} \operatorname{tr}(\underline{\underline{\varepsilon}})-3 \alpha_{\mathrm{m}} \mathrm{p}+\frac{\mathrm{C}_{\varepsilon}^{0}}{\mathrm{~T}_{0}} \tau
\end{gathered}
$$

Les sept paramètres indépendants sont, par exemple:

$\lambda$, $\mu$ coefficients de Lamé non drainés;

b. M paramètres de Biot:

$\alpha$ coefficient de dilatation linéique non drainé ; $\alpha$ coefficient de dilatation linéique drainé:

$\mathrm{C}_{e}^{\circ}$ chaleur volumique drainée à déformation constante.

Les autres paramètres intervenant dans les équations (8) à (10) se déduisent des sept précédents grâce aux formules suivantes:

$$
\begin{gathered}
3 K_{0}=3 \lambda_{0}+2 \mu \quad 3 K=3 \lambda+2 \mu \quad K_{0}=K-b^{2} M \\
\alpha K-\alpha_{0} K_{0}=\alpha_{m} b M
\end{gathered}
$$

\section{- Équations de couplage}

Le report des équations de comportement (8) et (9) respectivement dans les équations de diffusion (3) et (6), permet d'écrire les deux équations couplées fondamentales de notre problème linéarisé:

$$
\begin{gathered}
k \Delta p=\frac{1}{M} \frac{\partial p}{\partial t}-3 \alpha_{m} \frac{\partial \tau}{\partial t}+b \frac{\partial}{\partial t}(\operatorname{tr} \underline{\underline{\varepsilon}}) \\
K_{T} \Delta \tau=C_{\varepsilon}^{0} \frac{\partial \tau}{\partial t}-3 \alpha_{m} T_{0} \frac{\partial p}{\partial t}+3 \alpha_{0} K_{0} T_{0} \frac{\partial}{\partial t}(\operatorname{tr} \underset{\underline{\varepsilon}}{\underline{\varepsilon}})-r
\end{gathered}
$$

Ces équations ont la forme d'équations de diffusion simple (du type $\frac{\partial \mathrm{X}}{\partial \mathrm{t}}=\mathrm{k} \Delta \mathrm{X}$ où $\mathrm{k}$ est la diffusivité et $\Delta$ symbolise le laplacien) avec des termes de couplage supplémentaires.

Rappelons que dans notre cas, les termes de couplage dans l'équation thermique (14) sont négligeables.

\section{- Calcul des diffusivités}

Il est possible d'établir à partir de (13) et (14) des équations de diffusion simples portant respectivement sur $\Delta p$ et $\Delta \tau$. Pour cela suivons le raisonnement suivant:

* En tenant compte des identités mathématiques suivantes ( $\zeta$ est le déplacement):

$$
\begin{aligned}
& \underline{\operatorname{div}}(\operatorname{grad} \underline{\zeta})=\underline{\operatorname{grad}}(\operatorname{div} \underline{\zeta})-\underline{\operatorname{rot}}(\underline{\operatorname{rot}} \underline{\zeta}), \\
& \underline{\operatorname{div}}\left({ }^{\prime} \operatorname{grad} \underline{\underline{\zeta}}\right)=\underline{\operatorname{grad}}(\operatorname{div} \underline{\zeta}) .
\end{aligned}
$$

le report de la relation de comportement (8) dans l'équation d'équilibre (7), permet d'écrire :

$$
(3 \lambda+2 \mu) \underline{\operatorname{grad}(\operatorname{tr} \underline{\varepsilon})}-\mu \underline{\operatorname{rot}}(\underline{\operatorname{rot} \zeta} \underline{\underline{\zeta}})-\underline{\operatorname{grad}}\left(\frac{\mathrm{bM}}{\rho_{i 1}^{0} \mathrm{~g}} \mathrm{~m}+3 \alpha \mathrm{K} \tau\right)=0
$$

On peut remplacer la variable $m$ dans cette dernière équation par p grâce à (9). Il vient l'équation de Navier (utiliser également, pour simplifier, les relations (11) et (12) entre les paramètres):

$$
\left(\lambda_{0}+2 \mu\right) \underline{\operatorname{grad}}(\operatorname{tr} \underline{\varepsilon})-\mu \underline{\operatorname{rot}}(\underline{\operatorname{rot}} \underline{\xi})-\underline{\operatorname{grad}}\left(\mathrm{bp}+3 \alpha_{0} \mathrm{~K}_{0} \tau\right)=0
$$

* De façon à éliminer le rotationnel dans (15), il suffit de prendre la divergence de cette équation. On obtient facilement:

$$
\Delta\left(\left(\lambda_{0}+2 \mu\right) \operatorname{tr} \underline{\underline{\varepsilon}}-\mathrm{bp}-3 \alpha_{0} \mathrm{~K}_{0} \tau\right)=0
$$

On a ainsi établi une relation linéaire entre les laplaciens de $\operatorname{tr} \underline{\varepsilon}$, p et $\tau$.

* tr $\varepsilon$ intervient dans les deux équations de diffusion (13) et (14). Pour éliminer ces termes grâce à (16) et obtenir ainsi deux équations couplées portant sur les deux inconnues p et $\tau$ seulement, il suffit de prendre le laplacien des équations (13) et (14).

On trouve:

$$
\left(\begin{array}{ll}
\mathrm{d}_{\mathrm{m}} & \frac{\beta}{\mathrm{k}} \\
\frac{\beta \mathrm{T}_{0}}{\mathrm{~K}_{\mathrm{T}}} & \mathrm{d}_{\mathrm{s}}
\end{array}\right) \frac{\partial}{\partial t}\left(\begin{array}{c}
\Delta \mathrm{p} \\
\Delta \tau
\end{array}\right)=\Delta\left(\begin{array}{c}
\Delta \mathrm{p} \\
\Delta \tau
\end{array}\right)
$$

où l'on a posé, comme Dangla, 1994 :

$d_{m}=\frac{1}{k}\left(\frac{1}{M}+\frac{b^{2}}{\lambda_{0}+2 \mu}\right) d_{s}=\frac{T_{0}}{K_{T}}\left(\frac{C_{c}^{4}}{T_{0}}+\frac{9\left(\alpha_{n} K_{0}\right)^{2}}{\lambda_{0}+2 \mu}\right) \beta=3\left(\frac{b \alpha_{0} K_{0}}{\lambda_{0}+2 \mu}-\alpha_{m}\right)$

Dans (17), on a supposé, pour simplifier les calculs, que $\Delta r=0$.

Le coefficient de couplage $\beta$ s'écrit également:

$$
\beta=\frac{3 \alpha_{0} K_{0}}{b} k(1-\chi) d_{m}
$$

avec:

$$
\chi=\frac{\alpha K}{\alpha_{0} K_{0}} \frac{\lambda_{0}+2 \mu}{\lambda+2 \mu}
$$

Le paramètre adimensionnel $\chi$, qui «mesure» l'écart entre comportement drainé et comportement non drainé, a été introduit par Giraud, 1993 (voir aussi Giraud et Rousset, 1995).

Il permet de quantifier les couplages THM dans un milieu poreux sous l'effet d'un chargement purement thermique.

On trouvera dans les deux références citées plus haut des valeurs typiques de $\chi$ pour plusieurs géomatériaux poreux.

Pour les argiles profondes en particulier on passe de $\chi=1$ (c'est-à-dire $\beta=0$ ) qui correspond en fait à un milieu monophasique, à des valeurs de l'ordre de 10 pour les argiles plastiques, comme l'argile de Boom, pour lesquelles les couplages sont très importants.

(17) apparaît comme un système de diffusion couplé portant sur les variables $\Delta p$ et $\Delta \tau$ de la forme:

$$
\frac{\partial}{\partial \tau}\left(\begin{array}{l}
\Delta p \\
\Delta \tau
\end{array}\right)=\underline{A} \cdot \Delta\left(\begin{array}{l}
\Delta p \\
\Delta \tau
\end{array}\right)
$$

avec:

$$
\stackrel{\underline{A}}{ }^{-1}=\left(\begin{array}{cc}
d_{m} & \frac{\beta}{k} \\
\frac{\beta T_{0}}{K_{T}} & d_{s}
\end{array}\right)
$$

Nous appellerons les valeurs propres de la matrice de diffusion A qui apparaît dans (19), les coefficients de diffusion du problème.

Dans notre cas précis, les valeurs numériques du tableau de la figure 12 confirment que dans la deuxième équation du système (17), le terme $\frac{\beta \mathrm{T}_{\mathrm{D}}}{\mathrm{K}_{\mathrm{T}}} \frac{\partial}{\partial \mathrm{t}} \Delta \mathrm{p}$ peut être 
négligé et que dans l'expression $\mathrm{d}_{\mathrm{s}}$ donnée en (18) sous forme d'une somme, le premier terme de la somme est prépondérant devant le second.

Ainsi, le problème thermique est découplé en pratique et l'on écrit :

$$
\frac{\partial \tau}{\partial t}=k_{T} \Delta \tau
$$

où:

$$
\mathrm{k}_{\mathrm{T}}=\frac{1}{\mathrm{~d}_{\mathrm{s}}}=\frac{\mathrm{K}_{\mathrm{T}}}{\mathrm{C}_{\mathrm{E}}^{0}}
$$

est la diffusion thermique du problème posé.

Par abus de langage, on appellera diffusion hydraulique, et l'on notera $k_{h}$ la deuxième valeur propre de $\underline{\underline{A}}$

\section{- Application à l'argile de Boom}

Grâce aux données du tableau de la figure 12, pour l'argile de Boom, on trouve les valeurs de diffusion suivantes:

$$
\mathrm{k}_{\mathrm{T}}=6.10^{-7} \mathrm{~m}^{2} / \mathrm{s} \quad \mathrm{k}_{\mathrm{h}}=0,7.10^{-7} \mathrm{~m}^{2} / \mathrm{s}
$$

Les temps caractéristiques des diffusions hydraulique et thermique, pour un problème donné de dimension caractéristique a, sont donc:

$$
\tau_{\mathrm{T}}=\frac{\mathrm{a}^{2}}{\mathrm{k}_{\mathrm{T}}} \tau_{\mathrm{h}}=\frac{\mathrm{a}^{2}}{\mathrm{k}_{\mathrm{h}}}
$$

Le tableau suivant permet de retrouver les ordres de grandeurs des durées des phénomènes de diffusion données dans le texte.

Constantes de temps des phénomènes de diffusion

\begin{tabular}{l|c|c|c|}
\hline Problème & a & $\tau_{\text {f }}$ & $\tau_{\mathrm{b}}$ \\
Eprouvette & $0.1 \mathrm{~m}$ & 5 heures & 40 heures \\
Champ proche & $1 \mathrm{~m}$ & 20 jours & 160 jours \\
Champ lointain & $10 \mathrm{~m}$ & 5 ans & 40 ans \\
\hline
\end{tabular}

\section{Bibliographie}

Beaufays R., De Bruyn D., Moerkene K. Mine-by-test : a long term monitoring programme around an underground structure in Boom Clay, CEC report. Nuclear Science and Technology, EUP 15719 EN, 1994.

Bernaud D., Rousset G. - L'essai de soutênement à convergence contrôlée : résultats et modélisation. Int. Symp. Hard Soils - Soft rocks, Athens, 1993.

Bjot M.A. - General theory of three-dimensional consolidation. J. of App. Physics, 12, 1941. pp. 155-164.

Coussy 0. - Mécanique des millieux poreux, Éditions Technip, Paris, 1991.

Dangla P. - Analyse dimensionnelle, École de mécanique des milieux poreux. Aussois, distribué par le Comité Français de Mécanique des Roches, BRGM 45060 Orléans, 1994.

Djéran L. - Diffusions thermique et hydraulique dans une arqile soumise à un champ de température, thèse ENPC. Paris, 1991

Djèran I., Rousset G. - Contribution a l'étude des couplages thermo-hydromécaniques dans les arqiles. 7 th International Congress on Rock Mechanics, Aachen Germany, 16-21 sept. 1991.

Giraud A. - Couplages Thermo-HydroMécaniques dans les milieux poreux peu perméables : application aux argiles profondes, Thèse ENPC, Paris, 1993.
Giraud A., Picard J.-M., Rousset G. - Time dependant behavior of tunnels excavated in porous rock mass. Int. J. Rock. Mech. Min. Sci. \& Geomech. Abs.. vol. 30, n². 1993, pp. 1453-1459.

Giraud A., Rousset G. - Thermoelastic and thermoplastic response of a porous space submitted to a decaying heat source. Int J. for Num and Anal. Methods in Geomechanics, vol. 19, 1995 , pp. 475-495.

Giraud A. Rousset G. - Consolidation around a volumic spherical decaying heat source. Int. J. of Thermal Stresses. vol. 18, 1995, p. 513-536.

Horseman S.T., Winter M.G., Entwistle D.C. - Geotechnical characterization of Boom clay in relation to the disposal of radioactive waste, CEC report. Nuclear Science and Technologie, EUR 10987 EN, 1987.

Hueckel T.. Baldi G. - Thermoplasticity of saturated clays: Experimental constitutive study. Journal of Geotechnical Enqineering, vol. 116, 1990, n 12

Hueckel T., Borsetto M. Peano A. - Modelling of coupled thermo-elastoplastichydraulic response of clays subjected to nuclear waste heat, Numerical methods for transcient and coupled problems, John Willey and sons Ltd. 1987

Ould Amy M. Rousset G. - Modélisation numérique en thermo-poro-élastoplas- tícité d'un stockage souterrain de déchets radioactifs en milieu argileux saturé. Application au calcul du champ lointain. Revue Francaise de Géotechnique, $\mathrm{n}^{\circ} 69,1994, \mathrm{pp} .11-30$.

Picard J-M. Bazargan B. Rousset G. Essais thermo-hydro-mécaniques dans une argile profonde. CEC report. Nuclear Science and Technologv, EUR 15482 FR, 1994.

Picard J.-M. - Ecrouissage thermique des arqiles saturées: application au stockage de déchets radioactifs, thèse ENPC. Paris, 1995.

Rousset G. - Comportement mécanigue des argiles profondes, Thèse ENPC, Paris, 1988.

Rousset G., Bazargan B., Ouvry J.-F.. Bouilleau M. - Etude du comportement différé des argiles profondes. CEC report, Nuclear Science and Technology, EUR 14438 FR, 1988.

Wong H. - Comportement des galeries souterraines soumises à un chargement thermique, Thèse ENPC, Paris, 1994.

Wong H., Rousset G. - Tunnels profonds soumis à un chargement thermique. Revue Française de Géotechnique, $n^{\circ} 67$. 1994, pp. 13-32.

Wong H. Rousset G. - Thermoplastic behaviour of deep tunnels. Int. J. Thermal Stresses, vol. 17, 1994, $n^{\circ} 3$. 